\section{La relevancia del International Committee of Medical Journal Editors (ICMJE) para las publicaciones y la investigación médica}

\author{
HUMBERTO REYES B.
}

Editor, Revista Médica de Chile. Miembro, International Committee of Medical Journal Editors (ICMJE).

\section{The relevance of the International Committee of Medical Journal Editors (ICMJE) for medical publishing and research}

The International Committee of Medical Journal Editors is a leading independent institution providing guidance for the report of biomedical research and health related topics in medical journals. Established in 1978, it is currently constituted by editors of fourteen general medical journals from different countries, plus one representative for the US National Library of Medicine and one representative for the World Association of Biomedical Journal Editors. Since 1978 the Committee provides a document, originally named "Uniform Requirements...", "to help authors, editors, and others involved in peer review and biomedical publishing create and distribute accurate, clear, unbiased medical journal articles". This document has been updated several times and the last version was released in August 2013, now renamed "Recommendations for the Conduct, Reporting, Editing, and Publication of Scholarly Work in Medical Journals", available in www.icmje.org and citable as "ICMJE Recommendations". A vast proportion of medical journals, worldwide, have adopted these recommendations as rules. The ICMJE discusses and provides guidance on several relevant aspects including criteria on authorship, peer review, scientific misconduct, conflicts of interest, clinical trials registration, good editorial practices, the relations between editors and journal owners, the protection of individuals subject to medical research, the solvency of electronic publications, among others. The 2013 ICMJE Annual Meeting took place in Santiago, Chile, in November 4 and 5. The photograph shows attendants to the final session.

(Rev Med Chile 2014; 142: 79-83)

Key words: Committee membership; Editorial policies; Journalism, medical.
E n 1978 se reunió en Vancouver, Canadá, un grupo de editores de revistas médicas para elaborar un documento que guiara a los autores y a los editores, estandarizando el formato de los manuscritos médicos científicos, considerando un estilo propuesto por la National Library of Medicine de los Estados Unidos de Norteamérica cuyo Index Medicus, actualmente MEDLINE, es la principal base de datos para las publicaciones médicas. El documento resultante fue difundido en 1979, como "Uniform Requirements for Manuscripts Submitted to Biomedical Journals (URM)", con la autoría de los editores constituidos en el International Committee of Medical Journal Editors (ICMJE).

El documento "URM" ha tenido revisiones y reediciones (1997, 2003 y 2010), ampliando progresivamente su cobertura a temas más allá 
que el formato de los manuscritos, tales como la revisión por especialistas externos, los criterios de autoría, las situaciones de mala conducta científica, los conflictos de intereses, la protección de las personas que son objeto de una investigación, el registro de los ensayos clínicos en repositorios con acceso público en la Internet, las relaciones entre las entidades propietarias de las revistas y la independencia de los editores, las buenas prácticas editoriales, los requisitos de solvencia para las revistas electrónicas, y otros.

En agosto de 2013 se publicó la versión más reciente del documento y, para reflejar mejor el contenido y propósito actuales, su nombre cambió a "ICMJE Recommendations for the Conduct, Reporting, Editing and Publication of Scholarly Work in Medical Journals" (citable como "ICMJE Recommendations") ${ }^{1}$.

El presente artículo destacará algunos puntos de las "ICMJE Recommendations":

\section{Criterios que deben cumplir quienes figuren como autores de un manuscrito}

A los tres criterios enunciados en la versión previa "URM 2010" se ha agregado un cuarto criterio, definiendo que cada autor debe asumir la responsabilidad por la integridad del contenido total de un manuscrito.

Las "ICMJE Recommendations" establecen ahora que para figurar como autor de un manuscrito científico la persona debe cumplir TODOS los siguientes requisitos:

1. Aportó contribuciones sustanciales a la concepción o el diseño del estudio, o a la adquisición o interpretación de los datos para el estudio; Y

2. Participó en la redacción del manuscrito o en su revisión crítica, contribuyendo a su contenido intelectual; $Y$

3. Aprobó la versión final que será publicada; Y

4. Aceptó asumir responsabilidad por todos los aspectos del estudio, asegurando que se investigarán y resolverán apropiadamente las preguntas que pudieran surgir, relacionadas con cualquier parte del estudio.

Si surge una pregunta o duda sobre cualquier aspecto de un manuscrito es obligación de todos sus autores investigar y asegurar su resolución. No es aceptable que un autor responda "yo no participé en esa parte del trabajo y por eso me desentiendo de esta solicitud", ni tampoco "yo ya no pertenezco a la institución donde se hizo el estudio".

Frecuentemente los editores de revistas médicas reciben solicitudes de aclaraciones sobre cómo aplicar los criterios de autoría y de consejos para resolver dificultades surgidas al preparar un manuscrito o al enviarlo a publicación. La decisión de quien es o no autor depende del grupo de autores y no de los editores. Los editores deben exigir que quienes figuren como autores declaren cual fue su participación en el estudio respectivo y esa información puede ser publicada junto con el manuscrito.

Las "ICMJE Recommendations" son ahora más precisas para asignar formatos adecuados a la multi autoría en los trabajos colaborativos multicéntricos. Sin embargo, sigue siendo difícil entender que ocho o más personas figuren como autores en la comunicación de uno o dos casos clínicos.

\section{Definición de los ensayos clínicos}

El ICMJE define como ensayo clínico a todo proyecto de investigación que asigne personas en forma prospectiva a una intervención, con o sin su comparación concurrente o grupos controles, para estudiar la relación causa-efecto entre una intervención relacionada con salud y un "outcome" o efecto en salud. Las intervenciones relacionadas con salud son aquellas utilizadas para modificar un "outcome" biomédico o relacionado con salud; sus ejemplos incluyen fármacos, procedimientos quirúrgicos, dispositivos, prótesis, tratamientos conductuales, intervenciones dietéticas, intervenciones para mejorar la calidad de la salud y de los cuidados de la salud. Los "outcomes" en salud son cualquiera medición biomédica o relacionada con salud obtenida en pacientes o participantes, incluyendo mediciones farmacocinéticas y efectos adversos.

\section{El registro de los ensayos clínicos en repositorios con acceso público universal}

Desde el año 2008 las revistas que adhieren a las recomendaciones del ICMJE deben exigir que los ensayos clínicos hayan sido incorporados, antes de enrolar al primer paciente o participante, a un 
registro que cumpla con requisitos establecidos por la Organización Mundial de la Salud. Los registros que cumplen todos esos requisitos son reconocidos como "registros primarios", con validez internacional. Un modelo de registro primario se encuentra en http://clinicaltrials.gov

Los registros de ensayos clínicos exigen que los investigadores declaren la pregunta que quieren responder o la hipótesis por investigar, y definan los "outcomes" primarios y secundarios que pretenden obtener. Los editores, los revisores y los lectores doctos pueden comparar lo que se expresa en un manuscrito derivado de un proyecto de investigación con lo propuesto originalmente en ese proyecto. Pueden así verificar si el manuscrito se refiere adecuadamente al propósito inicial o a la hipótesis planteada, y si cumple con presentar y comentar sus resultados de acuerdo con los "outcomes" primarios y secundarios establecidos en el registro.

Además, los registros permiten acceder a información sobre estudios que pudieran no haber sido publicados porque tuvieron resultados negativos o porque se detectaron reacciones adversas que motivaron su interrupción.

\section{Declaración de potenciales conflictos de intereses}

La declaración de potenciales conflictos de intereses por los autores de un manuscrito científico contribuye a la transparencia de su contenido, permitiendo a los revisores y a los lectores juzgar el eventual peso de sesgos en la autoría. El ICMJE ofrece un formulario electrónico en www.icmje. org que los autores pueden adjuntar a su manuscrito para enviarlo a cualquier revista adscrita a este formato. Los autores archivan sus declaraciones en sus computadoras, las actualizan cuando fuere necesario y las pueden reutilizar para otros manuscritos enviados a la misma u otra revista. El formulario ha sido ensayado por muchas revistas $y$ autores, recibiendo observaciones que han permitido simplificarlo.

\section{La protección de las personas que son sujetos de investigación u observación y se incluyen en publicaciones}

Un tema ético con particular relevancia es la protección que se debe dar a los participantes en las investigaciones clínicas. La herramienta fundamental consiste en la aprobación del proyecto de investigación por Comités de Ética de la Investigación, algo que las revistas médicas deben exigir no sólo a los ensayos clínicos sino también a las investigaciones descriptivas y a las realizadas en animales de experimentación.

A ella se agregan ahora los requerimientos adoptados por la legislación de distintos países. En la Comunidad Europea y en Norteamérica se exige que toda persona que sea sujeto de una investigación clínica haya dado un consentimiento informado para ser incluida en una publicación, particularmente cuando en esa publicación pudiera revelarse, o sospecharse, la identidad de las personas estudiadas. Varias revistas médicas están ya exigiendo que las investigaciones clínicas cumplan este requisito. El ICMJE propondrá un documento cuyo formato pueda ser utilizado como guía con este efecto, reconociendo que deberá modificarse localmente según la legislación de un determinado país y el idioma nacional.

\section{La solvencia de las revistas electrónicas}

La publicación de revistas electrónicas prolifera universalmente, facilitando la difusión de la información científica e incluso dando paso a una discusión libre sobre las observaciones clínicas y las investigaciones formales. A consecuencia de ello los investigadores están siendo acosados por revistas nuevas, que los invitan a enviarles sus manuscritos, ofreciéndoles conseguir su revisión en plazos breves y una publicación rápida. Hay evidencias de que muchas revistas electrónicas no cumplen requisitos de solvencia editorial: la rapidez con que se aceptan los manuscritos hace dudar que hayan sido evaluados por revisores idóneos, lo que se refleja en que la publicación resultante tiene deficiencias notorias de fondo y forma; pero, el pago de derechos de publicación es un requisito que deben cumplir los autores, a veces antes de la aceptación del manuscrito. Esas revistas, calificadas como "predadoras", no están indizadas en las principales bases de datos, situación que suelen ignorar los autores y que los perjudica cuando se juzgan sus publicaciones.

El ICMJE, en conjunto con el Committee on Publication Ethics (COPE), la Open Access Scholarly Publishers Association (OASPA) y la World Associa- 
tion of Biomedical Editors (WAME) están preparando un documento que define los criterios para que una revista electrónica pueda ser considerada "creíble" y éticamente idónea. Este documento se pondrá a disposición de los autores y de las bases de datos internacionales.

\section{De Vancouver, 1978, a Santiago, 2013}

El "International Committee of Medical Journal Editors" es una organización independiente, relevante para el progreso científico universal, en particular en el área médica, lo que se refleja en que sus recomendaciones son respetadas como normas por la mayoría de las revistas médicas del mundo y contribuyen poderosamente a controlar la calidad, transparencia y confiabilidad de las publicaciones científicas médicas. La composición del comité se ha renovado periódicamente, manteniendo un número limitado de miembros que en su mayoría son editores de revistas médicas generales con gran prestigio. El comité tiene su sede administrativa en el American College of Physicians y su revista Annals of Internal Medicine, en Filadelfia. Los miembros se reúnen una vez al año, rotando las ciudades, pero se mantienen en contacto para consultas y discusiones a través del teléfono y el correo electrónico.

La Revista Médica de Chile adoptó en 1980 las indicaciones de los "URM" y en 1982 figuró como una de las 192 revistas que hasta ese momento suscribían el acuerdo, entre 263 publicaciones periódicas listadas en el Abridged Index Medicus ${ }^{2}$. Las Instrucciones a los Autores de la Revista son desde entonces una versión abreviada de los "URM", se ha reiterado a los autores de manuscritos que deben atenerse a los requisitos formales de ese documento, actualizado, y se han incorporado progresivamente otras variables, considerándolas como normas de publicación. Más allá de las formalidades, los editores han velado por la calidad de los manuscritos que publican, considerando su originalidad y la relevancia del contenido.

Desde 2010 la Revista exige que los autores de manuscritos adjunten una declaración de potenciales conflictos de intereses, utilizando el formulario universal del ICMJE. Una síntesis debe insertarse en la página de título de los manuscritos publicados y las declaraciones originales están disponibles para los lectores que las soliciten a la Revista. Al aceptar la revisión de un manuscrito, las personas invitadas deben declarar si tienen o no conflictos de intereses con los autores o con el contenido del manuscrito.

Nuestro país no cuenta todavía con un registro de ensayos clínicos que cumpla con los requisitos para ser aceptado internacionalmente como "Registro primario". Los editores de la Revista son parte activa en el proceso multi institucional que intenta resolver esta falencia.

La Revista Médica de Chile procura proteger el anonimato de las personas que son sujeto de investigación, particularmente en la presentación de casos clínicos. Sin embargo, para cumplir en todos sus aspectos con la nueva legislación nacional, debemos esperar que los legisladores consulten a las instituciones apropiadas (Academia Chilena de Medicina, Facultades de Medicina, Sociedades científicas médicas, Colegio Médico) para establecer una reglamentación apropiada para nuestra realidad social y cultural, evitando generar consecuencias que hagan inconfortable la investigación clínica.

Habiendo participado en un concurso abierto para editores y revistas médicas, en el año 2010 se incorporó a la Revista Médica de Chile -representada por su Editor jefe- como Miembro del $\mathrm{ICMJE}^{3}$. El Comité está constituido actualmente por los editores de catorce revistas médicas de Estados Unidos de Norteamérica, Canadá, Reino Unido, Holanda, Dinamarca, Noruega, Nueva Zelandia, Etiopía, China y Chile, más un representante de la U.S. National Library of Medicine, de los Estados Unidos de Norteamérica, y otro de la World Association of Biomedical Journal Editors. $\mathrm{Al}$ editor infrascrito le correspondió participar en las reuniones anuales sostenidas en 2010 en Queenstown, Nueva Zelanda; en 2011 en Ottawa, Canadá; en 2012 en Boston, EE UU de NA.

La reunión correspondiente al año 2013 se efectuó los días 4 y 5 de noviembre, en Santiago, siendo institución anfitriona la Sociedad Médica de Santiago-Sociedad Chilena de Medicina Interna, representada por el Dr. Guillermo Acuña Leiva. Los participantes en esta reunión fueron: Christine Laine, Annals of Internal Medicine; Darren Taichman (Secretario del Comité, de Annals of Internal Medicine); Fiona Godlee, British Medical Journal (BMJ); John Fletcher, Canadian Medical Association Journal (CMAJ); Mouyue Wang, Chinese Medical Journal (CMJ); Peter de Leeuw, Dutch Medical Journal; Howard Bauchner, Jour- 


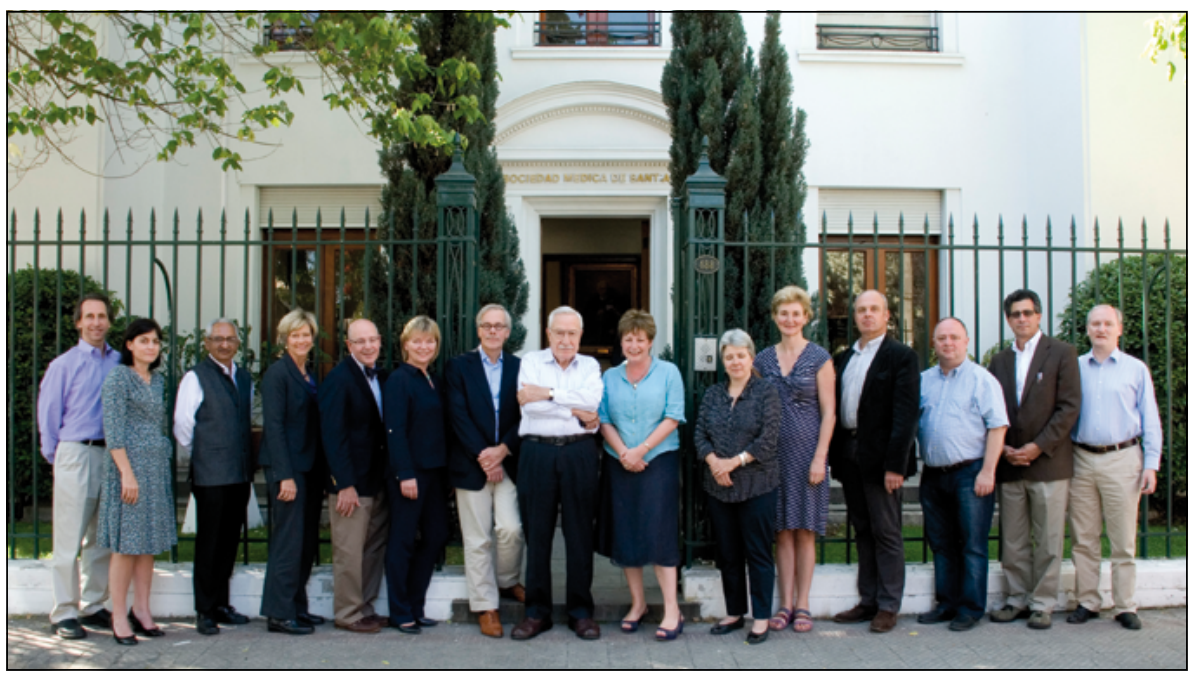

Participantes en el "2013 ICMJE Meeting" en Santiago, Chile, noviembre 4 y 5: Darren Taichman (Secretario Ejecutivo del ICMJE) y Christine Laine, Annals of Internal Medicine; Peush Sahni, World Association of Biomedical Journal Editors; Joyce Backus, National Library of Medicine; Jeffrey Drazen, New England Journal of Medicine; Charlotte Haug, Norwegian Medical Journal; Peter de Leeuw, Dutch Medical Journal; Humberto Reyes, Revista Médica de Chile; Astrid James, Lancet; Virginia Barbour, PLOS (Public Library of Science); Fiona Godlee, British Medical Journal; Frank Frizelle, New Zealand Journal of Medicine; Jacob Rosenberg, Journal of the Danish Medical Association; Howard Bauchner, JAMA (Journal of the American Medical Association); John Fletcher, Canadian Medical Association Journal. Ausente en la fotografía: Mouyue Wang, Chinese Medical Journal.

nal of the American Medical Association (JAMA); Jacob Rosenberg, Journal of the Danish Medical Association; Astrid James, Lancet; Jeffrey Drazen, New England Journal of Medicine (NEJM); Charlotte Haug, Norwegian Medical Journal; Frank Frizelle, New Zealand Journal of Medicine; Virginia Balbour, Public Library of Science (PLOS); Humberto Reyes, Revista Médica de Chile; Joyce Backus, National Library of Medicine (NLM); y Peush Sahni, World Association of Biomedical Journal Editors (WAME). La sesión final se realizó en el salón del Directorio de la Sociedad Médica de Santiago (ver Fotografía).

\section{Referencias}

1. International Committee of Medical Journal Editors. Recommendations for the Conduct, Reporting, Editing and Publication of Scholarly Work in Medical Journals. Publicado en agosto de 2013 y disponible en www.icmje. org

2. Goic A, Reyes H. Instrucciones a los autores de los trabajos sometidos a publicación en la Revista Médica de Chile (Editorial). Rev Med Chile 1986; 114: 617.

3. Reyes H. Revista Médica de Chile: Nuevo Miembro del "International Committee of Medical Journal Editors" (Editorial). Rev Med Chile 2010; 138: 665-8. 